Journal of Computer Science 6 (4): 386-391, 2010

ISSN 1549-3636

(C) 2010 Science Publications

\title{
A Performance Analysis of Modified Mid-Square and Mid-Product Techniques to Minimize the Redundancy for Retrieval of Database Records
}

\author{
${ }^{1,2}$ K. Meenakshi Sundaram, ${ }^{2}$ T. Santhanam, ${ }^{3}$ M. Saroja and ${ }^{4}$ C.P. Sumathi \\ ${ }^{1}$ Department of Computer Science, Erode Arts and Science College, Erode, India \\ ${ }^{2}$ Department of Computer Science, Dwaraka Doss Goverdhan Doss Vaishnav College, Chennai, India \\ ${ }^{3}$ Department of Electronics, Erode Arts and Science College, Erode, India \\ ${ }^{4}$ Department of Computer Science, Shrimathi Devkunvar Nanalal Bhatt Vaishnav College, Chennai, India
}

\begin{abstract}
Problem statement: An important tool in the field of education methodology is examination. As far as teaching-learning-evaluation process is concerned, the major task associated with the objective-type examination system is the administration of question paper setting. The lack of expertise and time are the major constraints that are encountered in the task of setting objective type question papers. During retrieval of records from a objective type question bank, redundancy may occur. To solve this problem, an approach needed to retrieve records from a database without redundancy. Approach: The task associated in generating the required collection of questions from a question bank, with minimal redundancy as far as possible in the retrieval of records from the question bank using mid-square and mid-product techniques for random number generation were discussed in this study. Results: A modified approach was identified and handled to generate random numbers and used to retrieve records from a database Conclusion: The suggested modified approach was more suitable for retrieving records from a database of even smaller size.
\end{abstract}

Key words: Minimizing redundancy, record retrieval, objective type question bank, mid-square technique, mid-product technique

\section{INTRODUCTION}

An important aspect of education process is examination. Teaching, learning and evaluation are the different components of education system. Evaluation is carried out at various stages in a number of ways. The teacher evaluates the performance of the students while teaching, at the end of the day, after completing the subject syllabus, at the end of the year. The response of the students makes evaluation as a continuous process and it has to be preplanned systematically and scientifically. The 1964-66 education commission report has pointed out that the examination reform has become crucial to all progress and it has to go hand in hand with the improvements in teaching. Radhakrishnan Commission, in their study has suggested the examination reforms as the key objective of the university education (AIU, 1994). The 1986 New Education policy lays more stress on the two major goals of examination reform programmes. One of them is the reliability and validity of examinations and the other one is making them as powerful instruments of teaching and learning. Examination system is useful in many ways. It not only helps the teacher to identify the weaker students but also in identifying the students who are good at the subjects. Further, it can help the teacher in suggesting some remedial measures to the weaker students. Examination is also quite useful to find out how much a student stands in a group. Moreover, it can be used to find the components, topics, units and subjects in which a student is strong and weak. Examination motivates the learner to be regular and attentive. Examination is an effective feedback mechanism to the teacher improving his instruction methods. It assists the teacher in identifying the relative strength of the students and to improve in the areas or topics where the students are lacking. Thus, examination is a tool in the self development of the individual

The examination process helps in developing a better curriculum, the areas in which all learners score the maximum marks, a topic which is too trivial and such topics may be excluded from the curriculum in the future. The most commonly used method of evaluation is through examinations. A standard question paper is to be set administered to all the students. The

Corresponding Author: T. Santhanam, Department of Computer Science, Dwaraka Doss Goverdhan Doss Vaishnav College, Chennai, India Tel: 91-044-9444169090 
advantages of this type of evaluation are, all the students are given the same set of questions, the best and worst scores can be easily determined. Question paper designed scientifically can be used as a powerful tool for examination not only in the education process but also for recruitments for jobs, to shortlist candidates from among a very large list of candidates. Thus, setting question papers scientifically and properly evaluating them may be the effective tools for an examination system.

Related works: A computer is a significant tool which must be a part of examination system. For the various tasks involved in the examination system numerous models have been developed so far. The various models surveyed are presented here. A computerized question banking system was discussed by Newbould and Massey (1976), described about the functions and characteristics of a computerized question banking system. It related to the process and requirements of the multiple-choice test development. The authors justify the use of multiple-choice questions alone, since the use of this type of test is increasing significantly and also recommend, choosing questions from a larger question bank. An approach was designed for the generation, administration and correction of multiple choice tests by Lira et al. (1990). Johnson and Maher (1982), describe the performance monitoring using a computerized question banking system.

Santhanam et al. (2008) has tried out to minimize the redundancy in the retrieval of records from question bank using Mid-square method. An attempt has been made to minimize the redundancy in the retrieval of records from question bank by applying Mid-Product method (Meenakshi et al., 2009). Positions of questions in the question bank are randomized and then it starts the test construction procedure. Mehrotra (2003) describes, a prototype knowledge based system for question paper setting was developed using artificial intelligence concepts. A comprehensive algorithm for this task cannot be used for an expert system. This is because of the fact that major portions of the problem domain of expert systems are invariably characterized by unstructured, not too formal knowledge. The algorithms used in the systems are generation of pseudo random number sequences and create randomly a large number of different tests. In the area of question paper setting, systems are being developed but setting question paper without human intervention has not been attempted. Since, syllabus is not uniform, framing syllabus should be attempted before setting the question paper. Syllabus and the question paper for a subject should be made independent of each other because of varying nature of the syllabus. A separate question bank has to be maintained, if the prescribed syllabus is separated by units of study. While creating and updating a database, the principles, reduce the redundancy and avoid the inconsistency to some extent must be followed (Date et al., 2009). Therefore, it is essential to develop a computer-aided objective type question paper system which permits the flexibility, with minimizing redundancies in the questions.

\section{MATERIALS AND METHODS}

Mid-square technique: This technique starts with an initial number or seed and then squared and the middle digits of this square become the random number after placement of the appropriate decimal. The middle digits are then squared to generate the second random number. The above process is repeated until the required number of random numbers is generated (Banks et al. 2009).

Example: Suppose that a sequence of four digit random numbers is needed:

$\mathrm{X}_{0}=5497$

$\mathrm{X}_{0}^{2}=(5497)^{2}=30217009 \Rightarrow \mathrm{X}_{1}=2170=\mathrm{R}_{1}$

$\mathrm{X}_{1}{ }^{2}=(2170)^{2}=04708900=>\mathrm{X}_{2}=7089=\mathrm{R}_{2}$

$\mathrm{X}_{2}^{2}=(7089)^{2}=50253921 \Rightarrow \mathrm{X}_{3}=2539=\mathrm{R}_{3}$

Mid-product technique: The mid-product technique starts by selecting two seeds $X_{0}$ and $X_{0}$ ' each containing the same number of digits D. Now, multiply $\mathrm{X}_{0}$ by $\mathrm{X}_{0}$, to get a number $\mathrm{U}_{\mathrm{i}}$. Set $\mathrm{X}_{1}$ equal to the middle $\mathrm{D}$ digits of $\mathrm{U}_{1}$ with the placement of appropriate decimals to obtain R1. Next multiply $\mathrm{X}_{1}$ by $\mathrm{X}_{0}$ to obtain $\mathrm{U}_{2}$, set $\mathrm{X}_{2}$ equal to the middle $\mathrm{D}$ digits of $\mathrm{U}_{2}$. Place the appropriate decimal to obtain the next random number $\mathrm{R}_{2}$. Repeat the above process until the required number of random numbers is generated (Banks et al., 2009). The following example illustrates this technique.

Example: Generate a sequence of four-digit random numbers with seeds:

$$
\begin{aligned}
& \mathrm{X}_{0}{ }^{\prime}=2938 \text { and } \mathrm{X}_{0}=7229 \\
& \mathrm{U}_{1}=\mathrm{X}_{0}{ }^{\prime} * \mathrm{X}_{0}=2938 * 7229=21238802=>\mathrm{X}_{1}=2388=\mathrm{R}_{1} \\
& \mathrm{U}_{2}=\mathrm{X}_{0} * \mathrm{X}_{1}=7229 * 2388=17262852=>\mathrm{X}_{2}=2628=\mathrm{R}_{2} \\
& \mathrm{U}_{3}=\mathrm{X}_{1} * \mathrm{X}_{2}=2388 * 2628=06275664=>\mathrm{X}_{3}=2756=\mathrm{R}_{3}
\end{aligned}
$$


Proposed approach: The following algorithm describes the steps to be followed while selecting the objective type question records from a question bank:

- Set the required number of records (questions)

- Generate the random number using Mid-square or Mid-product technique

- The records are selected based on the random number generated and grouped into one collection

- Sometimes, the records appropriate to the random number generated may not be satisfying the given constraints. To eliminate this problem, codes are assigned to the questions serially that are satisfying the constraints, having a range.

- Apply the following formula to get the converted sample so as to be within the range:

$$
\mathrm{R}=\mathrm{m} \text { MOD } n
$$

Where:

$\mathrm{m}=$ The random number generated by the midsquare or mid-product technique

$\mathrm{n}$ = The upper limit of the total number of records

$\mathrm{R}=$ The new converted sample

- Even after this, the system may have the repeated number of converted samples

- For instance, let us consider that there are 200 records satisfying the given constraints

- While generating a random number, the system generates 427 as a random number

- It exceeds the upper limit 200. Hence, 27, the remainder of 427/200 is the new converted sample and the system selects the 27 th record

- After few selections, if the system generates 827 as random number, the converted sample is the same 27 th record, which is selected already

- To avoid this selection of repeated records, the actual number is also stored along with converted sample

- The system checks the new random number with the previous one (here comparison between 827 and 427)

- If it is same, the system gets new seed, since degeneracy occurs in random number generation

- If it is different, the system ignores the random number and generates next new random number based on the mid-square technique or mid-product technique (here 27 is ignored)

- This process eliminates the restriction on having a total number of records satisfying the given constraints as large

\section{RESULTS AND DISCUSSION}

Mid-square technique application:

Case-A1: In this case, the system sets a question paper consisting of 10 questions that are selected with the seed value 5678 out of 382 questions using modified approach of mid-square technique for random number generation (Table 1).

Case-A2: In this case, the system sets a question paper consisting of 10 questions which are selected with the seed value 5678 out of 368 records using modified approach of mid-square technique for random number generation (Table 2).

It can be noted that, though the seed value and method of random number generation is same as the case-A.1, this case selects different questions, since the total number of questions satisfying the constraints are different.

Case-A3: In this scenario, the system sets a question paper consisting of 10 questions with the seed value 5678 out of 21 questions using modified approach of mid-square technique for random number generation (Table 3).

Case-A4: Here, the system sets a question paper consisting of 10 questions with the seed value 5678 out of 16 questions using modified approach of mid-square technique for random number generation (Table 4).

\begin{tabular}{lcc}
\multicolumn{3}{l}{ Table 1: Selection of 10 records from 382 records for the seed 5678} \\
\hline Random number & Converted sample & Sample considered \\
\hline 2396 & 104 & 104 \\
7408 & 150 & 150 \\
8784 & 380 & 380 \\
1586 & 58 & 58 \\
5153 & 187 & 187 \\
5534 & 186 & 186 \\
6251 & 139 & 139 \\
750 & 368 & 368 \\
5625 & 277 & 277 \\
6406 & 294 & 294 \\
\hline
\end{tabular}

Table 2: Selection of 10 records from 368 records for the seed 5678

\begin{tabular}{lcc}
\hline Random number & Converted sample & Sample considered \\
\hline 2396 & 188 & 188 \\
7408 & 48 & 48 \\
8784 & 320 & 320 \\
1586 & 114 & 114 \\
5153 & 1 & 1 \\
5534 & 14 & 14 \\
6251 & 363 & 363 \\
750 & 14 & $* * *$ \\
5625 & 105 & 105 \\
6406 & 150 & 150 \\
368 & 0 & 0 \\
***: Repeated converted sample-ignored &
\end{tabular}


Table 3: Selection of 10 records from 21 records for the seed 5678

\begin{tabular}{lcc}
\hline Random number & Converted sample & Sample considered \\
\hline 2396 & 2 & 2 \\
7408 & 16 & 16 \\
8784 & 6 & 6 \\
1586 & 11 & 11 \\
5153 & 8 & 8 \\
5534 & 11 & $* * *$ \\
6251 & 14 & 14 \\
750 & 15 & 15 \\
5625 & 18 & 18 \\
6406 & 1 & 1 \\
368 & 11 & $* * *$ \\
1354 & 10 & 10 \\
\hline$* *$ Repeated converted sample-ignored
\end{tabular}

Table 4: Selection of 10 records from 16 records for the seed 5678

\begin{tabular}{lcc}
\hline Random number & Converted sample & Sample considered \\
\hline 2396 & 12 & 12 \\
7408 & 0 & 0 \\
8784 & 0 & $* * *$ \\
1586 & 2 & 2 \\
5153 & 1 & 1 \\
5534 & 14 & 14 \\
6251 & 11 & 11 \\
750 & 14 & $* * *$ \\
5625 & 9 & 9 \\
6406 & 6 & 6 \\
368 & 0 & $* * *$ \\
1354 & 10 & 10 \\
8333 & 13 & 13 \\
\hline
\end{tabular}

***: Repeated converted sample-ignored

Table 5: Selection of 10 records from 200 records for the seeds 5678, 2345

\begin{tabular}{lll}
\hline Random number & Converted sample & Sample considered \\
\hline 3149 & 149 & 149 \\
3844 & 44 & 44 \\
1047 & 47 & 47 \\
246 & 46 & 46 \\
2575 & 175 & 175 \\
6334 & 134 & 134 \\
3100 & 100 & 100 \\
6354 & 154 & 154 \\
6974 & 174 & 174 \\
3127 & 127 & 127 \\
\hline
\end{tabular}

\section{Mid-product technique application:}

Case-B1: In case-B1, the system sets a question paper consisting of 10 questions which are selected with the seeds 5678,2345 out of 200 questions using modified approach of mid- product technique for random number generation (Table 5).

Case-B2: Here, the system sets a question paper consisting of 10 questions which are selected with the seeds 5678, 2345 out of 160 records using modified approach of mid-product technique for random number generation (Table 6).
Table 6: Selection of 10 records from 160 records for the seeds 5678 ,

\begin{tabular}{|c|c|c|}
\hline Random number & Converted sample & Sample considered \\
\hline 3149 & 109 & 109 \\
\hline 3844 & 4 & 4 \\
\hline 1047 & 87 & 87 \\
\hline 246 & 86 & 86 \\
\hline 2575 & 15 & 15 \\
\hline 6334 & 94 & 94 \\
\hline 3100 & 60 & 60 \\
\hline 6354 & 114 & 114 \\
\hline 6974 & 94 & $* * *$ \\
\hline 3127 & 87 & $* * *$ \\
\hline 8076 & 76 & 76 \\
\hline 2536 & 136 & 136 \\
\hline
\end{tabular}

Table 7: Selection of 10 records from 20 records for the seeds 5678 ,

\begin{tabular}{lcc}
\multicolumn{1}{c}{2345} & & \\
\hline Random number & Converted sample & Sample considered \\
\hline 3149 & 9 & 9 \\
3844 & 4 & 4 \\
1047 & 7 & 7 \\
246 & 6 & 6 \\
2575 & 15 & 15 \\
6334 & 14 & 14 \\
3100 & 0 & 0 \\
6354 & 14 & $* * *$ \\
6974 & 14 & $* * *$ \\
3127 & 7 & 16 \\
8076 & 16 & $* * *$ \\
2536 & 16 & $* * *$ \\
4807 & 7 & 5 \\
1905 & 5 & 13 \\
1573 & 13 & \\
\hline
\end{tabular}

***: Repeated converted sample-ignored

It can be noted that, though the seed value and technique of random number generation is same as the Case-B1 this case selects different questions, since the total number of questions satisfying the constraints are different.

Case-B3: Case-B3 depicts, the system sets a question paper consisting of 10 questions with the seeds 5678, 2345 out of 20 questions using modified approach of mid-product technique for random number generation (Table 7).

Case-B4: The system sets a question paper consisting of 10 questions with the seeds 5678, 2345 out of 15 questions using modified approach of mid- product technique for random number generation in Case-B4 (Table 8).

The performance of the above two discussed techniques are shown in the Table 9 and 10 and plotted in the graphs Fig. 1 and 2. 
Table 8: Selection of 10 records from 15 records for the seeds 5678, 2345

\begin{tabular}{lcc}
\hline Random number & Converted sample & Sample considered \\
\hline 3149 & 14 & 14 \\
3844 & 4 & 4 \\
1047 & 12 & 12 \\
246 & 6 & 6 \\
2575 & 10 & 10 \\
6334 & 4 & $* * *$ \\
3100 & 10 & $* * *$ \\
6354 & 9 & 9 \\
6974 & 14 & $* * *$ \\
3127 & 7 & 7 \\
8076 & 6 & $* * *$ \\
2536 & 1 & 1 \\
4807 & 7 & $* * *$ \\
1905 & 0 & 0 \\
1573 & 13 & 13 \\
***: Repeated converted sample-ignored
\end{tabular}

Table 9: Performance analysis while retrieving 10 records using midsquare technique

\begin{tabular}{lllll}
\hline & & & \multicolumn{2}{l}{ Record retrieved using } \\
& & & $\begin{array}{l}\text { Mid-square } \\
\text { technique }\end{array}$ & $\begin{array}{l}\text { Modified mid- } \\
\text { square technique }\end{array}$ \\
Case & Seed value & Max rec & te--- \\
I & 5678 & 382 & 10 & 10 \\
II & 5678 & 368 & 7 & 10 \\
III & 5678 & 21 & 5 & 10 \\
IV & 5678 & 16 & 2 & 10 \\
\hline
\end{tabular}

Table 10: Performance analysis while retrieving 10 records using mid-product technique

\begin{tabular}{|c|c|c|c|c|}
\hline \multirow[b]{2}{*}{ Case } & \multirow[b]{2}{*}{ Seed value } & \multirow[b]{2}{*}{ Max rec } & \multicolumn{2}{|c|}{ Record retrieved using } \\
\hline & & & $\begin{array}{l}\text { Mid-product } \\
\text { technique }\end{array}$ & $\begin{array}{l}\text { Modified mid- } \\
\text { product technique }\end{array}$ \\
\hline I & 5678,2345 & 200 & 10 & 10 \\
\hline II & 5678,2345 & 160 & 8 & 10 \\
\hline III & 5678,2345 & 20 & 7 & 10 \\
\hline IV & 5678,2345 & 15 & 5 & 10 \\
\hline
\end{tabular}

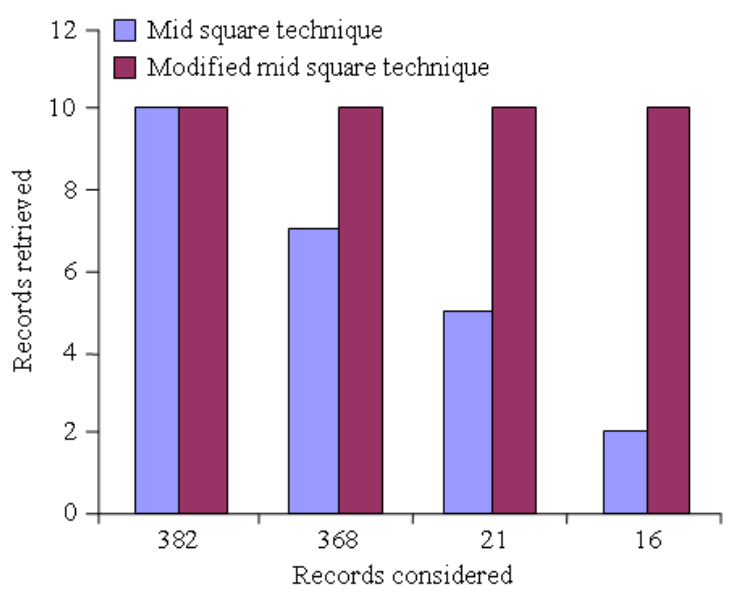

Fig. 1: Performance comparison of mid-square technique with modified mid-square technique

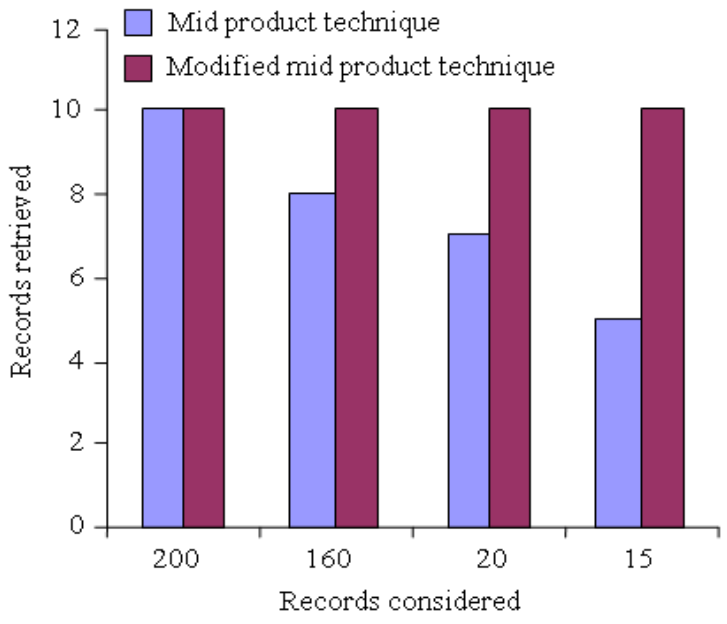

Fig. 2: Performance comparison of mid-product technique with modified mid-product technique

\section{CONCLUSION}

It is very evident from Table 9 and 10, Fig. 1 and 2, regarding $100 \%$ retrieval of records without redundancy is possible using the modified approach than the midsquare technique and mid-product technique. Also, it justifies that the modified approach is appropriately suitable to retrieve the records even with small collection of database records eliminating redundancy. This study discussed the issues associated with the various tasks of objective type question paper setting in an automated fashion. While maintaining the question bank, the records are inserted through the key values. Sometimes it is possible that the question bank may contain some questions which appear to be different but conveying the same meaning. Future research can be focused on, to remove such kind of redundancy in the question bank and also the possibility of exploring the other statistical techniques or tools to generate random numbers.

\section{REFERENCES}

AIU., 1994. Staff Development in Indian UniversitiesMonograph on Question Banking for the Universities. AIU, ISBN: 81-7520-011-1, pp: 45-48.

Banks, J., J.S. Carson, B.L. Nelson and D.M. Nicol, 2009. Discrete Event System Simulation. 5th Edn., PHI, ISBN: 13: 9780136062127, pp: 252-254.

Date, C.J., A. Kannan and S. Swamynathan, 2009. An Introduction to Database Systems. 8th Edn., Pearson Education, ISBN: 978-81-7758-556-8, pp: 18-19. 
Johnson, S. and B. Maher, 1982. Monitoring science performance using computerized question banking system. Br. J. Educ. Technol., 13: 97-106. DOI: 10.1111/j.1467-535.1982.tb00433.x

Lira, P., M. Bronfman and J. Eyzaguirre, 1990. Multi test II: A program for the generation, correction and analysis of multiple choice tests. IEEE Trans. Educ., 33: 320-325. DOI: 10.1109/13.61083

Meenakshi, S.K., T. Santhanam, M. Saroja and C.P. Sumathi, 2009. Minimizing redundancy in the retrieval of records from question bank using mid-product method. Proceedings of the International Conference on Emerging Trends in Computing, Kamarajar Jan. 8-10, College of Engineering and Technology, Virudhunagar, India, pp: 69-72.
Mehrotra, D., 2003. Impact of Information Technology on Quality Education. Scribd, pp: 8-9. http://www.scribd.com/doc/21369241

Newbould, C.A. and A.J. Massey, 1976. A computerized item banking system. Br. J. Educ. Technol., 8: 114-123. DOI: 10.1111/j.14678535.1977.tb00205.x

Santhanam, T., M. Saroja, C.P. Sumathi and S.K. Meenakshi, 2008. Minimizing redundancy in the retrieval of records from question bank using mid-square method. Proceedings of the International Conference on Mathematics and Computer Science, July 25-26, Loyola College, Chennai, pp: 137-142. 